Voix et Images

voixetimages

\title{
Dialogue autour de Ferron
}

\section{Chantal Gamache}

Volume 16, numéro 3 (48), printemps 1991

François Charron

URI : https://id.erudit.org/iderudit/200928ar

DOI : https://doi.org/10.7202/200928ar

Aller au sommaire du numéro

Éditeur(s)

Université du Québec à Montréal

\section{ISSN}

0318-9201 (imprimé)

1705-933X (numérique)

Découvrir la revue

Citer cet article

Gamache, C. (1991). Dialogue autour de Ferron. Voix et Images, 16(3), 532-535.

https://doi.org/10.7202/200928ar d'utilisation que vous pouvez consulter en ligne.

https://apropos.erudit.org/fr/usagers/politique-dutilisation/ 


\section{Dialogue autour de Ferron}

\section{par Chantal Gamache, Université de Montréal}

Le rapprochement et l'interférence de plus en plus diversifiés des cultures nous confrontent au commerce des langages et des valeurs. Le problème de la traduction devient incontournable: Bien plus qu'un simple passage d'un code à l'autre, que la recherche de l'équivalence formelle, elle est ouverture à l'aútre, à la différence. Aventure impossible, elle saisit ce qu'il y a d'insaisissable dans les textes d'autrui, et qui pourtant modelise la forme dans les contraintes d'un certain matériau linguistique. Le traducteur, empreint de sa propre historicité, solidaire du "tout de l'œuvre" originale et de son auteur auxquels il doit fidélité, se projette néanmoins comme médiateur vers le lecteur, dans les limites d'un autre code linguistique. Traductrice des œuvres de Jacques Ferron, Betty Bednarsky nous propose un ensemble d'essais critiques sur son expérience de traduction difficile et passionnante ${ }^{1}$.

Cette publication, issue d'une thèse de doctorat, ne porte pourtant pas les marques de la rigidité scolaire qui caractérisent souvent ce genre. Elle n'en a ni la lourdeur rhétorique et stylistique ni le 
même rapport à l'officiel. Cela ne me semble pas dépendre de la seule révision du texte de la thèse pour satisfaire aux exigences de l'édition. La construction textuelle d'ensemble, la souplesse, la vivacité de l'écriture et surtout, la nature même du discours critique qui s'y construit conferent à cette étude sur la traduction un statut particulier. C'est ce qu'affirme l'enthousiasme de Jean Marcel Paquette dans la préface:

Et pourtant, ici, nous voilà transportés dans quelque chose qui ressemble à l'implacable réalité du songe, aux justes limites de l'accessible tant l'écriture $y$ est d'un charme dense, aux frontières de l'intraduisible tant l'argumentation est souple.

Ces qualités posent peut-être ce texte aux justes limites de l'accessible mais, cependant, elles convoquent l'interaction du lecteur empathique. Comment traverser cet ouvrage de Betty Bednarsky sans constamment être pris de l'envie de l'interroger tout à coup, d'intervenir sur une question posée ou sous-entendue ou sur un point de vue; d'échanger, de partager, de discuter, de glisser avec l'auteure dans une dynamique dialogique de la lecture.

C'est bien l'effet que produisent ces réflexions sur la traduction. Non seulement elles définissent cette dernière comme un geste actif de lecture et puis d'écriture, mais aussi comme un lieu privilégié de "l'expérience de l'autre». Ces rapports d'altérité se déploient dans tous les sens. Élément axiologique de l'analyse que l'auteure façonne du processus de traduction, et plus spécifiquement des œuvres de Jacques Ferron, la notion d'altérité travaille son propre discours dans sa projection de lecture. La nature de ses considerations et la forme de leur mise en texte nous constituent en lecteurs interagissants, devenus nous-mêmes virtuels producteurs de discours. Ces propos s'inscrivent dans cette relation, dans ce dialogue déjà lancé.

L'ensemble des considérations de l'auteure analyse en profondeur ses attitudes de traductrice de l'œúvre de Ferron. Là où j'aimerais apporter quelques commentaires, ou plutôt là où j'aimerais échanger avec elle, c'est sur nos visions respectives de l'œuvre de Ferron. Nous observons les mêmes phénomènes. Cependant, notre lecture en est peut-être un peu différente. J'aimerais soulever, ici, un lieu de discussion possible puisqu'il est au centre de l'activité de traduction. De plus, il m'apparaît soulever des questions fondamentales pour une lecture de Ferron. Il s'agit de la présence de la langue d'accueil transformée par la langue de départ dans le texte original.

Le passage des écrits de Ferron en langue anglaise se heurte à une difficulté majeure: celle de la traduction des mots anglais appartenant aux discours sociaux québécois et dont l'écriture se soumet dans le texte à la graphie reproduisant de façon "malicieuse" les sonorités propres à l'oralité française du Québec. Ces intrusions dans 
le texte portent en elles toute l'intensité des rapports des deux univers linguistiques. Mais je ne crois pas que l'on doive isoler ce phénomène discursif bien qu'il soit particulier. Il s'inscrit dans une articulation culturelle située à un niveau supérieur plus englobant, celui de l'énonciation, sur le plan du dialogisme interne du texte. Et là, le mot se définit dans un rapport à la norme.

Ces mots anglais francisés ne sont pas les seules présences étrangères remarquées chez Ferron. Les mots anglais non francisés, les archaïsmes, les mots latins posés dans le texte comme des ornements, sont inclus dans une structure syntaxique française correcte ainsi valorisée. Dans cette nouvelle mise en situation des langages sociaux, les différences se trouvent ainsi nivelées dans un discours unifiant. Elles ne sont que l'écho de la multiplicité sociale des langages.

De plus, ajoutons que pour les Québécois francophones, la langue anglaise est porteuse de luttes pour la survie, contre l'asservissement et la domination, non seulement grosse du poids des générations de Québécois qui ont dû s'y soumettre pour travailler, pour consommer, mais aussi pleine de rêves, de vacances, de bonheur, de fortune. C'est ce que projette le fameux neveurmagne de Ferron qui, en fait, tel que reproduit dans sa sonorité, appartient aux discours culturels québécois hors texte. Ce patois du mythique Survenant, personnage libre, audacieux et sans attaches souffrantes du roman de Germaine Guèvremont, fut sur toutes les lèvres. Et c'est dans son appropriation sonore francophone qu'on le prononça. C'est cela que nous entendons, dans les textes de Ferron, dilué, baigné dans un français correct, conforme à la norme. Comme tous les autres mots, les mots anglais francisés ornent les textes de Ferron. Ils témoignent d'une grande sensibilité à la diversité des langages, mais ils sont d'une certaine manière la confirmation de l'aspect normatif du code dominant français des collèges classiques.

Ces propos ne contredisent pas ceux de Betty Bednarski. Au contraire, je crois qu'ils doublent la difficulté de traduction. Comment trouver dans une autre langue l'équivalent de ces rapports qui rendent justice au texte original ? Entreprise impossible. L'objectivité de la traduction est impensable. Le texte traduit est l'autre texte d'une vision autre sur l'autre.

Je ne crois donc pas, comme Betty Bednarski, que la langue devienne implicitement le sujet du texte. Elle en est le lieu d'exercice avec tout ce qu'elle contient d'histoire et de luttes de valeurs. Le sujet du texte se dévoile dans ses enjeux de l'énonciation, dans la mise en rapport de langages. Le véritable sujet du texte est l'insaisissable du texte, et qu'il faut pourtant traduire.

Comme les mots, les noms propres me semblent des parures tirées directement de la réalité mythique de la culture québécoise. Constituent-ils vraiment des personnages avec tout ce que cela 
comporte d'épaisseur, de configuration? J'en doute. Ou bien, livrés subitement comme ils se donnent dans les contes, très proches des formes qu'ils prennent dans la culture orale, ou bien, exilés dans le texte sans forme intratextuelle autre que celle construite dans la mythologie culturelle hors-texte, les noms propres de personnes chez Ferron alimentent le rapport à la norme.

Betty Bednarski travaille tous ces problèmes en profondeur et de façon très personnalisée, ainsi que plusieurs autres aspects de la traduction. Elle passe en revue diverses formes de lecture: celle propre à la traduction, la lecture pédagogique et la lecture critique ainsi que les relations qu'elles ont entre elles. Puis, elle traite du problème de l'altérité à partir de son expérience spécifique de la traduction de Ferron. Finalement, elle conclut par un essai de passage de la vie à l'ouvre et de l'ouvre à la vie. L'ouvrage de Mikhaïl Bakhtine, Esthétique de la création verbale, lui a servi de fondement théorique. Ce choix me semble tout à fait pertinent puisque Bakhtine fut particulièrement intéressé par la vie et les rapports des langages, par le dialogisme intérieur des textes, celui qui les constitue, et par le dialogisme externe aux textes, celui dans lequel ils sont inclus. L'ouvrage de Betty Bednarski en est tout empreint.

1 Betty Bednarski, Autour de Ferron. Littérature, traduction, altérité, Toronto, GREF, 1989. 